\title{
EMG-Based Neuromuscular Modeling with Full Physiological Dynamics and Its Comparison with Modified Hill Model
}

\author{
Mitsuhiro Hayashibe, David Guiraud, Philippe Poignet
}

\begin{abstract}
EMG-based muscle model has many applications in human-machine interface and rehabilitation robotics. For the muscular force estimation, so-called Hill-type model has been used in most of the cases. It has already shown its promising performance, however it is known as a phenomenological model considering only macroscopic physiology. In this paper, we discuss EMG-force estimation with the full physiology based muscle model in voluntary contraction. In addition to Hill macroscopic representation, a microscopic physiology description as stated by Huxley and Zahalak is integrated. It has significant meaning to realize the same kind of EMGforce estimation with multiscale physiology based model not with a phenomenological Hill model, because it brings the understanding of the internal biophysical dynamics and new insights about neuromuscular activations.
\end{abstract}

\section{INTRODUCTION}

Any human movement is produced by muscular and skeletal systems controlled by the nervous system. Thanks to sports science and biomechanics research, the dynamics of the musculoskeletal system has been revealed by many experimental trials. However, neural aspects and muscle activation in neuromuscular system should be still intensively analyzed and modeled from microscopic scale to macroscopic scale as the pathway from neural command. Thus, neuromuscular modeling is quite important for neuroscience to understand how limb movements are controlled smoothly and effectively. It has also significant meanings for the clinical application concerning spasticity induced by stroke or cerebral palsy. Then, quantitative analysis between neural activity and muscle force can contribute to the design of Functional Electrical Stimulation (FES) for paralyzed muscles and myoelectrical limb control framework [1], and also to the design of robotic prosthetic limb and exoskeleton. The general musculoskeletal model of whole body and its dynamics computation method were established and available [2][3]. In this paper, we focus on developing EMG-based muscle model with microscopic physiology view in the activated muscle.

EMG-based models have already been used among many works to estimate torques around the joints [7]. Most muscle models are based on phenomenological model derived from Hill's classic work [5] and well summarized by Zajac [6]. Hill-type model has been used for a long time. Many studies have shown the promising result of EMG-driven musculoskeletal model to predict human joint moments [4]. For more physiologically detailed model, a microscopic model was designed by Huxley [9] and Zahalak [10]. Here, we try

The authors are with INRIA Sophia-Antipolis -DEMAR Project and LIRMM, UMR5506 CNRS UM2, 161 Rue Ada - 34392 Montpellier Cedex 5, France hayashibe, guiraud,poignet@lirmm.fr to realize EMG-force estimation with both microscopic and macroscopic physiology based model in voluntary contraction. It is known that force estimation by Hill-type model is promising. However, it would be meaningful to realize the similar EMG-force estimation with the full physiology based model not only with a phenomenological Hill model. Using common EMG data of isometric muscle contraction, the force estimation results are shown by modified Hill model and new physiology based approach. Its comparison and the discussion are provided.

\section{HILL-TYPE MUSCLE MODEL}

The transformation from EMG to muscle activation is an essential process in classical force prediction. Especially in Hill-type models, it is very dominant process because the estimated muscle force is assumed to be proportional to the muscle activation. Here, we describe typical steps to perform the transform. One should keep in mind that most researchers use a subset of the described method.

\section{A. EMG Processing}

We summarize the employed EMG processing method. For the detail, you can refer to the literature [4][7].

1) high-pass filtering of the raw EMG using zero-lag 4th Butterworth filter $(30 \mathrm{~Hz})$ to remove movement artifact

2) wave rectification

3) low-pass filtering with a $2-10 \mathrm{~Hz}$ cut-off frequency

4) normalization with the peak of Maximum Voluntary Contraction (MVC)

The normalized, rectified, filtered EMG is referred as $e(t)$. There exists a time delay for the muscle activation. The process of transforming $e(t)$ into neural activation $p(t)$, is called activation dynamics. When a muscle fiber is activated by a single Action Potential (AP), the muscle generates a twitch response. This response can be well represented by a critically damped linear second-order differential system. Its recursive discrete filter can be obtained as $p(t)=\gamma e(t-d)-$ $\beta_{1} p(t-1)-\beta_{2} p(t-2)$, where $d$ is the electromechanical delay and $\gamma, \beta_{1}$ and $\beta_{2}$ are the coefficients that define the secondorder dynamics. To realize a positive stable solution, a set of constraints was employed, i.e. $\beta_{1}=C_{1}+C_{2}, \beta_{2}=C_{1} C_{2}$ where $\left|C_{1}\right|<1,\left|C_{2}\right|<1$. In addition, the unit gain of this filter should be maintained by ensuring $\gamma-\beta_{1}-\beta_{2}=1$.

\section{B. Modification for Nonlinear Neural to Muscle Activation}

Many researchers assume that the aboved $p(t)$ is a reasonable approximation of muscle activation. However, nonlinear relationship has been reported between individual muscle 
EMG and joint moment for some muscles especially at lower forces [8]. In studies on single motor units, multiple APs cause multiple twitch responses. If the time between APs decreases, the twitches start to merge into each other and the muscle force increases steadily. However, at high frequency the twitches get closer to tetanus, where no further force is produced even if the frequency increases. This means there is a nonlinear relationship between frequency and force for single motor units.

Thus, nonlinearization from neural activation $p(t)$ to muscle activation $a(t)$ should be considered. As the simple and adequate solution, Lloyd [4] proposed following formulation:

$$
a(t)=\frac{e^{A p(t)}-1}{e^{A}-1}
$$

where $A$ is the nonlinear shape factor allowed to vary between -3 and 0 , with $A=-3$ being highly exponential and $A=0$ being linear.

\section{Hill-type Contraction Dynamics}

The muscle-tendon unit is modeled as a contractile element in series with an elastic tendon as in Fig. 1. The Hilltype muscle model is used to estimate the force $F_{c}(t)$ that can be generated by the contractile element with general form of the function by

$$
F_{c}(t)=a(t) f_{l}\left(\varepsilon_{c}\right) f_{v}\left(\dot{\varepsilon}_{c}\right) F_{0}^{m}
$$

where $\varepsilon_{c}$ is the strain of contractile element, $f_{l}\left(\varepsilon_{c}\right)$ and $f_{v}\left(\dot{\varepsilon}_{c}\right)$ are the normalized force-length and force-velocity relationships respectively. $F_{0}^{m}$ is the maximum isometric muscle force. $\phi$ is the pennation angle between the tendon and the muscle fibers. In this paper, only concentric contraction is considered, thus parallel elastic element is not introduced. The force length relationship shows a Gaussian distribution around the optimal length. In every time step, fiber velocity should be solved and muscle fiber length can be calculated by forward integration using Runge-Kutta algorithm. Since the value for $\varepsilon_{c}$ has changed, the calculation should continue iteratively until the end of input time series of $a(t)$.

Muscle tendon parameters were adopted from Delp [13]. The optimal lengths of contractile element $\left(L_{c 0}\right)$ are $3 \mathrm{~cm}$ for soleus and $5.1 \mathrm{~cm}$ for gastrocnemius. The tendon slack lengths $\left(L_{t 0}\right)$ are $26.8 \mathrm{~cm}$ for soleus and $40 \mathrm{~cm}$ for gastrocnemius. For gastrocnemius, parameters are averages of two heads (med/lat). $F_{0}^{m}$ and pennation angle $\phi$ are also obtained from this reference, but final result is normalized by maximum contraction, thus the effect of these parameters can be neglected.

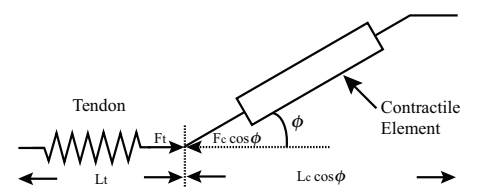

Fig. 1. Typical muscle-tendon macroscopic model.

\section{FULL PHYSIOLOGICAL MUSCLE MODEL}

The theory of microscopic physiological muscle model is based on the two-state cross-bridge model of Huxley [9] and the distribution-moment model of Zahalak [10]. Here, it is summarized briefly.

\section{A. Sarcomere scale}

All the sarcomeres are assumed to be identical, and the deformation of both sarcomere and muscle scale is proportional. If $S$ is the sarcomere length, we can write $\left(S-S_{0}\right) / S_{0}=\left(L_{c}-L_{c 0}\right) / L_{c 0}=\varepsilon_{c}$.

Huxley proposed that a cross-bridge between actine filaments and myosine heads could exist in two biochemical states, attached and detached states. He postulated that one myosine head could attach to only one actine site. Then, the dynamics of the fraction $n(y, t)$ of the attached cross bridges is given by

$$
\frac{\partial n}{\partial t}+\frac{S_{0}}{h} \dot{\varepsilon}_{c} \frac{\partial n}{\partial y}=f(y, t)[1-n(y, t)]-g(y, t) n(y, t)
$$

where $h$ is the maximum elongation of the myosine spring, $y$ the normalized distance between actine binding site and myosine head. $n(y, t)$ is a distribution function representing the fraction of attached cross bridges relative to the normalized position $y . S_{0} \dot{\varepsilon}_{C}$ represents the velocity of the actine filament relative to the myosine filament. $f$ and $g$ denote the rate functions of attachment and detachment respectively.

The chemical input was introduced by Bestel [11]. It proposed that cross bridge rates depend on the relative velocity between actine and myosine. The higher the velocity is, the greater the probability to break bridges is. We define the chemical input $u(t)$ as follows:

$$
\begin{aligned}
& u(t)=\Pi_{c}(t) U_{c}+\left(1-\Pi_{c}(t)\right) U_{r} \\
& \Pi_{c}(t)=1 \text { during contraction, } 0 \text { else } \\
& (f+g)(y, t)=u(t)+\left|\dot{\varepsilon}_{c}\right|
\end{aligned}
$$

$U_{c}$ and $U_{r}$ are the level of chemical kinetics under contraction and relaxation phases respectively.

Stiffness and force generated by a muscle sarcomere can be obtained by computing the first and second moment of the distribution $n(y, t)$.

\section{B. Myofiber and muscle unit scale}

The maximum available cross bridges could vary depending on the relative length of the contractile element. It is known as the force-length relationship $\left(f_{l}\left(\varepsilon_{c}\right)\right)$. Contrary to Hill model, we take into account this relation at the microscopic scale [12].

Let's define $k_{c}$ and $F_{c}$ as stiffness and force for the whole contractile element, and $N$ the number of all MUs. The number of recruited fibers is written as $\alpha N$ using recruitment ratio $\alpha$. Considering that each fiber is composed of identical sarcormeres in series, finally the desired model of contractile element is a set of differential equations as below:

$$
\begin{aligned}
& \dot{k}_{c}=-\left(u+\left|\dot{\varepsilon}_{c}\right|\right) k_{c}+\alpha k_{m} \Pi_{c}(t) U_{c} \\
& \dot{F}_{c}=-\left(u+\left|\dot{\varepsilon}_{c}\right|\right) F_{c}+\alpha F_{m} \Pi_{c}(t) U_{c}+k_{c} L_{c 0} \dot{\varepsilon}_{c}
\end{aligned}
$$



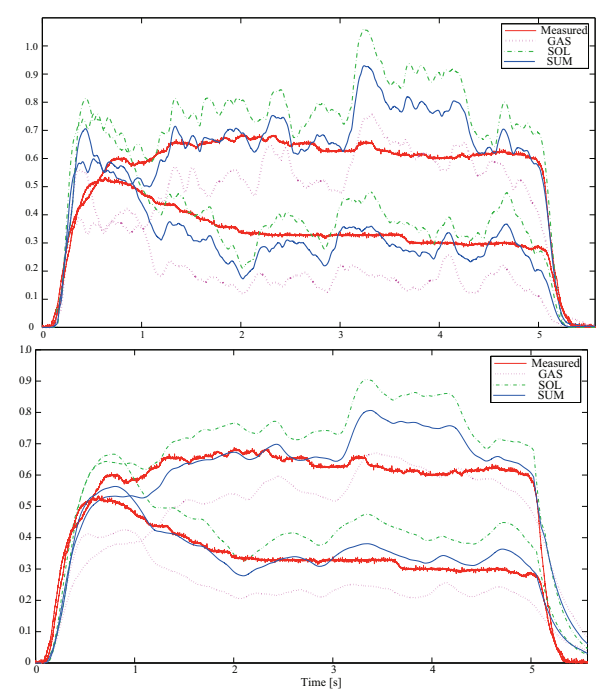

Fig. 2. Normalized estimated torques (red:measured, magenta:GAS, green:SOL, blue:SUM) for $30 \%$ and $70 \%$ of MVC by modified Hill model (top) and by full physiology based model (bottom).

where $k_{m}=S_{0} N k_{0} f_{l}\left(\varepsilon_{c}\right) / L_{c 0}, F_{m}=N k_{0} h f_{l}\left(\varepsilon_{c}\right) / 2 . k_{0}$ $\left(\mathrm{Nm}^{-1}\right)$ is the maximum stiffness obtained when all the available bridges are attached.

For the macroscopic representation, the same configuration in Fig. 1 is used including muscle tendon parameters. Only the contractile element is replaced with the above equations.

\section{RESULTS}

Two healthy subjects were seated on a chair with their right foot fixed on a Biodex dynamometer. The torque around ankle joint was measured when it is voluntarily generated for the extension. For EMG measurements, bipolar surface $\mathrm{Ag} / \mathrm{AgCl}$-electrodes were placed on the muscle belly of the medial Gastrocnemius (GAS) and Soleus (SOL).

Here, isometric torque was estimated only from EMG signals by the modified Hill model and the proposed physiology based muscle model. The predicted torque was compared to the measured torque by Biodex system. For this trial, we make the comparison for the normalized torque against the one in maximum voluntary contraction (MVC), therefore the absolute value of moment arm can be omitted. However, we still need to know the ratio of the contribution among both muscles of GAS and SOL for the resultant moment. The moment arm was estimated from the Hawkins [14] from the joint angle in the measured condition. The contribution ratio was calculated using the literature values of Delp [13] considering pennation angle. The resultant ratio is GAS 0.41 vs SOL 0.59. The SUM in the shown graphs is plotted using the ratio as the sum of two muscles.

For the new model, the rectified EMG was low-pass filtered with $30 \mathrm{~Hz}$ cut-off frequency at the third process in Section II.A. Then, chemical input $u(t)$ was created by thresholding the extracted EMG signals as shown in Fig. 3. The thresholding can be assumed as muscle cell's allor-nothing response to AP. Muscle contraction is initiated by an AP along the muscle fiber membrane, which causes calcium releases that induce the contraction process when
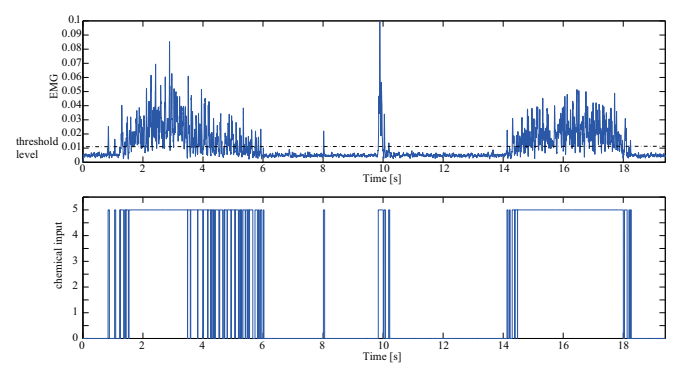

Fig. 3. Generation of chemical input. Top: filtered rectified EMG signal, bottom: generated chemical input by thresholding for SOL.

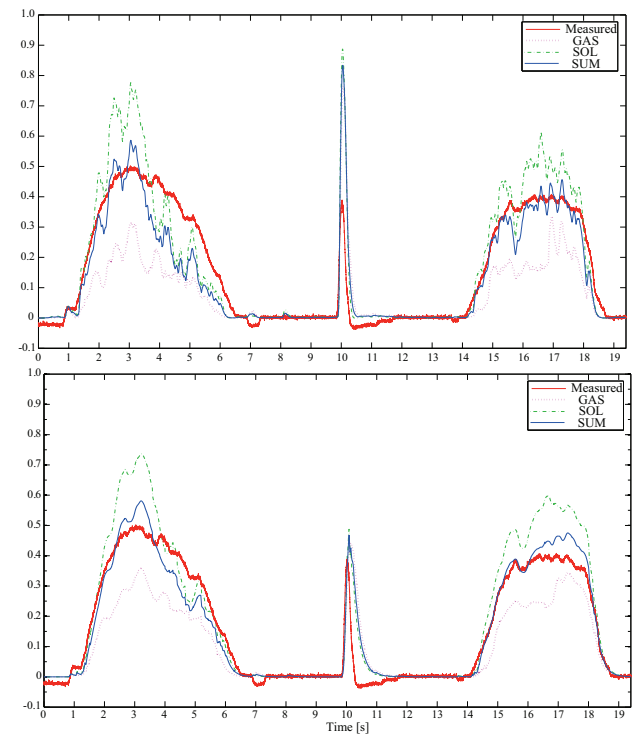

Fig. 4. Normalized estimated torques (red:measured, magenta:GAS, green:SOL, blue:SUM) for random contraction by modified Hill model (top) and by full physiology based model (bottom).

the concentration goes above a threshold and is sustained till the concentration goes down this threshold again. For the recruitment $\alpha$, normalized $p(t)$ in Section II.A was used.

The generated input command $u(t)$ was given to the contractile element of physiological model and the active stiffness $k_{c}$ and the muscle force $F_{c}$ were computed through Eq.5. In Fig. 2, normalized estimated torques for $30 \%$ and $70 \%$ of MVC by modified Hill model and physiology based model are shown. In the modified Hill, RMS errors between the measured and the estimated are 0.0988 for $70 \%, 0.0718$ for $30 \%$. In the proposed model, RMS errors are 0.0857 for $70 \%, 0.0363$ for $30 \%$.

In order to confirm the estimation ability both for shortterm contraction and long-term contraction, the result which includes two types of contractions is presented in Fig. 4. The chemical input in Fig. 3 corresponds to this result. The obtained RMS errors are given in Table I for both contraction types and by both estimation approaches.

TABLE I

RMS ERRORS BETWEEN THE MEASURED AND THE ESTIMATED

\begin{tabular}{cccccc}
\hline \multirow{2}{*}{ Subject } & \multicolumn{2}{c}{ Modified Hill } & & \multicolumn{2}{c}{ Full Physiology } \\
\cline { 2 - 3 } \cline { 5 - 6 } & short term & long term & & short term & long term \\
\hline \hline 1 & 0.0519 & 0.0523 & & 0.0313 & 0.0420 \\
2 & 0.1015 & 0.0823 & & 0.0705 & 0.0557 \\
\hline
\end{tabular}




\section{DISCUSSION}

The aim of the proposed full physiology model is not to improve the estimation precision. Hill-type muscle is a phenomenological model based on experimental facts with no link to the microscopic physiology. Even if the estimation performances are equivalent, it is meaningful to understand and capture the muscle dynamics with more detailed representation. In this paper, similar force estimation could be obtained with the full physiology based model compared to Hill estimation using voluntary EMG. If we take a look at the result of Hill model as in Fig. 4, we can find the larger error for the estimation of short-term contraction. The reason of the error can be considered as follows: the signal measured in EMG is the summation of the APs of all different motor units (MU). So even in the short-term tetanic contraction, the amplitude of EMG itself is the same level as in longterm tetanic contraction. However, the resultant force of short-term contraction is actually much less than the one of long-term contraction. It means that there is somewhat time hysteresis regarding the neural command. In Hill approach, muscle activation $a(t)$ is proportional to the resultant force. It does not include an effect of time hysteresis in contraction, therefore Hill model can not well estimate both the short and long-term contractions at the same time with one choice of certain cut-off frequency in EMG low-pass filtering. In the proposed approach, the derivative of the contraction force is directly given by the neural command and it brings time hysteresis in force generation. The choice of threshold level is not so sensitive to the input generation. At least for shortterm contraction, it can be said that the proposed model can improve the estimation precision. It is interesting to see this kind of effect along with the introduction of Huxley crossbridge representation.

In addition, it is known that there is a nonlinear relationship between frequency during contraction and force for single motor units. In modified Hill model, this frequency dependency is tried to be offset only by the nonlinear conversion as in Section II.B. This nonlinearization was proposed recently to modify classical Hill model. Originally this process has not been introduced in so-called Hill model. In fact, this modification brings much better estimation especially at lower forces. Even with the modification, it is not a time function so that it still can not correspond to the different speeds of muscle contraction. In the proposed model, the nonlinear activation property is internally integrated. However, the modification in Hill model is external from the contraction dynamics. The integrative expression is also advantage for the proposed method. It can be also verified that the full physiology model integrates the forcevelocity relationship naturally from the consideration of actin-myosin cross bridge in Eq. 5.

\section{CONCLUSION}

In this paper we have presented a method that allows to estimate the muscle force from EMG signal with a full physiology based model with a link to underlying microscopic filament dynamics. The results highlight the feasibility of the torque estimation by the full physiology model and its comparison with modified Hill model using same EMGs. For the common parameters between two approaches, the same value was used. The proposed method features:

- a novel physiologically detailed model for EMG-force estimation instead of a phenomenological Hill-type muscle model,

- the estimation improvement especially for lower activation and short-term contraction with the natural integration of the frequency property of neural activation.

The summation of electrical activity created by each MU appears in EMG. Strictly, EMG signals should be decomposed into constituent MUAPs to obtain the map of MU firings. However, the generation of chemical command by thresholding EMG can at least capture the fact that the higher the firing rate of MUs is, the larger the fused force of twitches is. Future work will focus on increasing the number of tests and the further interpretation of neuromuscular system.

\section{ACKNOWLEDGMENTS}

We would like to thank A. Varray and M. Papaiordanidou at Univ. of Montpellier I for their help at the experiments.

\section{REFERENCES}

[1] D. Guiraud, T. Stieglitz, K.P. Koch, J.L. Divoux, P. Rabischong, ”An implantable neuroprosthesis for standing and walking in paraplegia: 5-year patient follow-up", J. Neural Eng., vol. 3, pp 268-275, 2006.

[2] S.L. Delp, F.C. Anderson et al, "OpenSim: Open-Source Software to Create and Analyze Dynamic Simulations of Movement", IEEE Trans. on Biomedical Engineering, vol. 54, no. 11, pp 1940-1950, 2007.

[3] Y. Nakamura, K. Yamane, Y. Fujita, and I. Suzuki, "Somatosensory Computation for Man-Machine Interface From Motion-Capture Data and Musculoskeletal Human Model", IEEE Trans. on Robotics, vol. 21, no. 1, pp 58-66, 2005.

[4] D.G. Lloyd and T.F. Bessier, "An emg-driven musculoskeletal model to estimate muscle forces and knee joint moment in vivo", Journal of Biomechanics, no. 36, pp. 765-776, 2003.

[5] A.V. Hill, "The heat of shortening and the dynamic constants in muscle", Proceeding of the royal society, London, Sre. B, vol. 126, pp. 136-195, 1938.

[6] F.E. Zajac "Muscle and tendon: properties, models, scaling and application to biomechanics and motor control". CRC Critic. Rev. in Biomed. Eng. 17: 359-411, 1989.

[7] T.S. Buchanan, D.G. Lloyd, K. Manal and T.F. Bessier, "Neuromusculoskeletal modeling: estimation of muscle forces and joint moments and movements from measurements of neural command", Journal of Applied Biomechanics, no. 20, pp. 367-395, 2004.

[8] K. Manal, T.S. Buchanan, "A one-parameter neural activation to muscle activation model", Journal of Biomechanics, vol. 36, pp. 11971202, 2003.

[9] A.F. Huxley, Muscle structure and theories of contraction. Progress in Biophysics and Biophysical Chemistry, vol. 7, pp. 255-318, 1957.

[10] G.I. Zahalak, A distribution-moment approximation for kinetic theories of muscular contraction. Mathematical Biosciences, vol. 55, pp. 89-114, 1981.

[11] J. Bestel, M. Sorine, A differential model of muscle contraction and applications. In schloessmann Seminar on Mathematical Models in Biology, Chemistry and Physics, Max Plank Society, Bad Lausick, Germany, May 19-23, 2000.

[12] H. El Makssoud, D. Guiraud, P. Poignet, Mathematical muscle model for Functional Electrical Stimulation control strategies. Proc. IEEE/Int. Conf. on Robotics and Automation, pp. 1282-1287, 2004.

[13] S.L. Delp, "Surgery simulation: a computer graphics system to analyze and design musculoskeletal reconstructions of the lower limb", Dissertation, Stanford University, CA, USA, 1990.

[14] D. Hawkins and M. Hull, "A method for determining lower extremity muscle-tendon lengths during flexion/extension movements", Journal of Biomechanics, vol. 23, pp. 487-494, 1990. 